\title{
Is nano too big?
}

\author{
Walter Mier • John Babich • Uwe Haberkorn
}

Published online: 3 October 2013

(C) The Author(s) 2013. This article is published with open access at Springerlink.com

The possibility to utilize nanotechnology for drug development has led to widespread interest and funding opportunities. The public perception of the field of nanoscale science originally referred to technologies dealing with nanomechanics, nanomaterials, nanooptics and nanoelectronics, but increasingly anticipates progress in nanomedicine. Today's molecular imaging possibilities are ideal for visualizing the pharmacokinetics of the effectors used in new treatment strategies such as gene therapy [1] and therapeutic vaccination [2]. With the possibility to visualize and the salient, concomitant treatment, nuclear medicine has the greatest potential for use in the development of innovative treatment strategies. Consequently, the eventual applications of nanosized tracers are of the greatest interest for our discipline.

Nanoparticles can be strictly defined as microscopic particles with at least one dimension less than $100 \mathrm{~nm}$. Nanoparticles used for pharmaceutical applications are normally above the renal exclusion limit (approximately $50 \mathrm{kDa}$ ) but small enough to pass through all blood capillaries. Together with the components required to provide specific functions (Fig. 1), targeted nanoparticles reach a few hundred nanometres in size (the size of the smallest bacteria), intermediate between large proteins (e.g. antibodies with a hydrodynamic radius of approximately $15 \mathrm{~nm}$ ) and microspheres (particles in the micrometre range, typically 10 to $200 \mu \mathrm{m}$ ). A distinction has to be made between microspheres and nanoparticles. Microspheres have gained increasing importance for two reasons. First, they can be used in the embolization of fine capillaries. Following locoregional administration into the blood vessels

W. Mier $\cdot$ U. Haberkorn $(\bowtie)$

Department of Nuclear Medicine, University Hospital Heidelberg, INF 400, 69120 Heidelberg, Germany

e-mail: uwe.haberkorn@med.uni-heidelberg.de

J. Babich

Department of Radiology, Cornell University, New York, USA supplying a tumour, microspheres accumulate in the tumour tissue. Radioembolization using ${ }^{90}$ Y-labelled microspheres such as SIR-Spheres ${ }^{\circledR}$ and TheraSpheres ${ }^{\circledR}$ can be used for the treatment of patients with unresectable hepatocellular carcinoma [3]. Second, they can be used in the location of the sentinel lymph node in the management of patients with early breast cancer. The sentinel node extracts radiolabelled particles from the lymph and can be intraoperatively detected using a hand-held gamma probe [4].

At first glance nanoparticles have the potential to greatly extend the current repertoire of therapeutic applications by providing the means to transport various cargos. In nuclear medicine applications this includes a choice of radionuclides for either diagnostic or therapeutic applications. Targeting of the particles can be achieved by modifiers readily chosen from the large repertoire of targeting molecules, such as peptides and antibodies. A large variety of nanoparticle delivery systems such as polymeric nanoparticles, liposomes, dendrimers, polymerosomes and inorganic particles have been described. The most prominent compounds are encapsulated cytotoxic drugs, such as DOXIL ${ }^{\circledR}$, PEGylated liposomes containing the topoisomerase inhibitor doxorubicin. However, PEGylated liposomal doxorubicin is still used far less than free doxorubicin as it causes dose-limiting side effects, differing from those of doxorubicin, notably palmoplantar erythrodysaesthesia (handfoot syndrome) [5].

Unfortunately, there are serious shortcomings in the clinical application of nanoparticles. Nanoparticles have a propensity to adsorb serum proteins such as opsonin causing their marking out for immediate destruction by the immune system. Biodistribution studies of unmodified nanoparticles have shown their rapid accumulation in the liver, spleen and bone marrow (reticuloendothelial system). Therefore, a major proportion of the particles are trapped by these normal organs and are not available for the target, i.e. tumour, tissue. While this problem can be avoided by coating with polyethylene glycol 


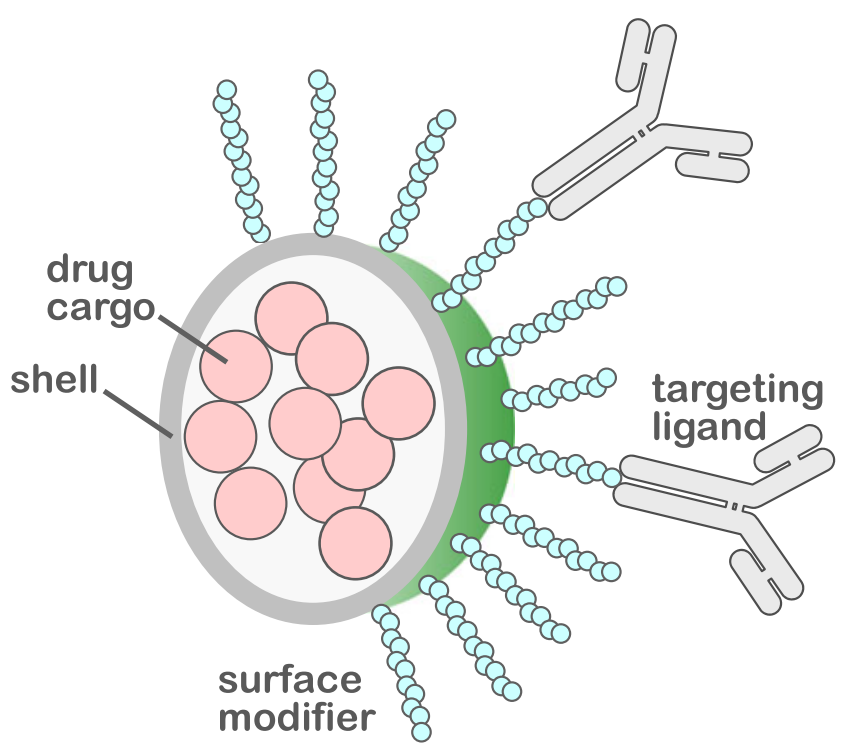

Fig. 1 Schematic illustration of the structure of a nanoparticle comprising the components presumed to enable targeted drug delivery

[6], it should be born in mind that any coating chosen to suppress recognition by the immune system is inevitably associated with interference with specific binding.

The lack of specificity of a naked nanoparticle has been approached by coupling the nanoparticles with targeting molecules such as antibodies, receptor affine peptides, folate receptor ligands or bisphosphonates on their surface [7]. However, the number of potential targeting molecules is limited to molecules that are not influenced by conjugation to particles. The strategy to direct the accumulation to a specific target whilst limiting nonspecific accumulation faces two problems: (1) the affinity of the targeting molecule is influenced by the large particle to which it is attached, and (2) the biodistribution of the small molecule/peptide/antibody is dominated by the large particle. All modifications required to facilitate targeting and biocompatibility, however, increase the size of the particle, and such increase is detrimental to the quality of the pharmaceutical which generally decreases with increasing size [8].

It is possible that nanoparticles can be transported through the interendothelial junctions which are enlarged in tumours. However, the likelihood that large constructs can penetrate deeply into tissue, and make their way back from sites where no specific interaction takes place can be estimated from a comparison with oxygen. The permanent oxygen shortage in large solid tumours reveals the enormous diffusion barrier to be overcome. The diffusion constants of $\mathrm{O}_{2}$ and macromolecules differ by many orders of magnitude. Tumours show a poorly organized vascular architecture and compression of blood and lymphatic vessels by cancer cells. In addition to hypoxia, this creates increased interstitial fluid pressure which can slow down the movement of molecules within the tumour and limits the delivery of drugs to cells located far from functioning blood vessels [9]. Mobility is therefore a main factor defining the performance of a tracer in an organism expressing a target at a defined specificity. Antibodies are an instructive example of this. Their large size counteracts their excellent properties and binding affinity. However, antibodies have the advantage of outstanding biocompatibility and therefore attain exceptional circulation times. This increases the probability of constructive interaction and thus compensates for their lower mobility.

Most discussions regarding the interaction of targeted molecules neglect the fact that the specific interaction will take place at the surface of the tumour cells and consequently far away from the bloodstream (Fig. 2). In many cases the proliferation of tumour cells leads to a cellular population that is at a considerable distance $(>100 \mu \mathrm{m})$ from vessels where the microenvironment is dictated by hypoxia and reduced $\mathrm{pH}$. This area also shows reduced vascular density. As a function of their size, all drugs suffer from a steep concentration gradient which decreases with increasing distance from the surrounding blood vessels [10].As a logical result clinical application of nanoparticles should be considered impossible. It is argued that nanocarriers can penetrate the tumour vasculature through its leaky endothelium and, in this way, accumulate in solid tumours; this is referred to as the enhanced permeation and retention (EPR) effect (Fig. 3). A simple calculation reveals that this effect cannot result in the uptake of significant amounts of a drug. For example, an injection of a hypothetical dose of $1 \mathrm{~g}$ of articles would compete for uptake with approximately $300 \mathrm{~g}$ of serum protein. An assumed capillary volume of $1 \mathrm{ml}$ in a tumour of $10 \mathrm{~cm}^{3}$ will accommodate a total of approximately $50 \mathrm{mg}$ of macromolecules, corresponding to $0.16 \mathrm{mg}$ of particles. It is therefore clear why the EPR effect can only be demonstrated in animal models

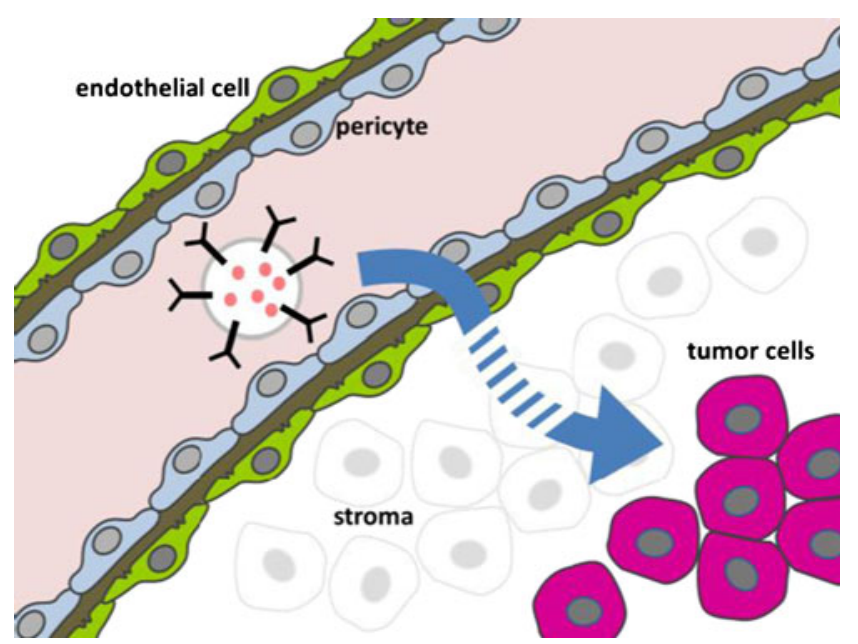

Fig. 2 Schematic illustration of the extravasation and distance to be bridged by targeted particles. Passage through the endothelial cell layer and migration over distances of the order of $100 \mu \mathrm{m}$ are required to reach tissue in which the particles might be retained by specific interaction 


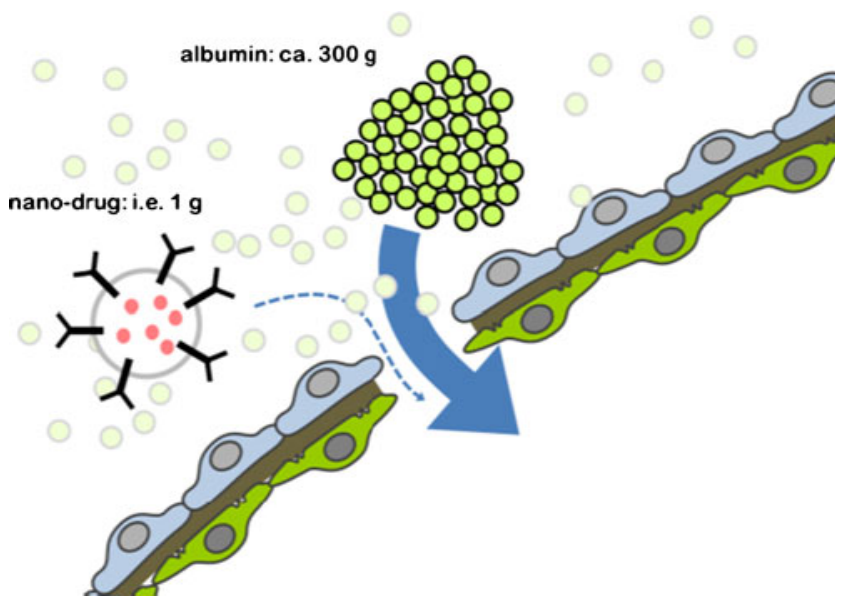

Fig. 3 Schematic illustration of extravasation at sites of disturbed microvasculature, the basis for the enhanced permeation and retention effect considered to be active in the selective uptake of nanoparticles. It is reasonable to consider that all macromolecules compete in this process, with a substantial disadvantage for nanoparticles because of their number and diffusion rate

with artificial tumours grown within days to reach a significant fraction of the whole animal weight [11], and not in clinical situations that physicians are confronted with in tumours in patients.

The main idea behind most nanosized drug platforms relies on their potential to encapsulate the cargo and thereby shield it during transport to the site of action, and preventing its action prior to arrival. Generally, this does not apply to radiopharmaceuticals as their effect - the radiation — cannot be shielded in the particle.

Finally, it has to be born in mind that the defined production of nanoparticles that fulfil the requirements for clinical use is extremely demanding. At present, it is already difficult to meet the regulatory requirements for small molecules. It is therefore hard to know whether it would be possible to consistently produce such complex products which vary in size, degree of loading, core composition, the number and site of modifications attached and many other issues such as the functionality of the labelling sites and the targeting moieties.

In conclusion, the size of nanoparticles will prevent their use as drugs. Attempts should therefore be focused on small molecules that possess the mobility required to exploit their specificity.
Open Access This article is distributed under the terms of the Creative Commons Attribution License which permits any use, distribution, and reproduction in any medium, provided the original author(s) and the source are credited.

\section{References}

1. Penuelas I, Haberkorn U, Yaghoubi S, Gambhir SS. Gene therapy imaging in patients for oncological applications. Eur J Nucl Med Mol Imaging. 2005;32 Suppl 2:S384-403. doi:10.1007/s00259-0051928-3.

2. Muthuswamy R, Mueller-Berghaus J, Haberkorn U, Reinhart TA, Schadendorf D, Kalinski P. PGE(2) transiently enhances DC expression of CCR7 but inhibits the ability of DCs to produce CCL19 and attract naive T cells. Blood. 2010;116:1454-9. doi:10.1182/blood2009-12-258038.

3. Sangro B, Carpanese L, Cianni R, Golfieri R, Gasparini D, Ezziddin S, et al. Survival after yttrium-90 resin microsphere radioembolization of hepatocellular carcinoma across Barcelona clinic liver cancer stages: a European evaluation. Hepatology. 2011;54:868-78. doi:10.1002/hep. 24451.

4. Lovrics PJ, Cornacchi SD, Vora R, Goldsmith CH, Kahnamoui K. Systematic review of radioguided surgery for non-palpable breast cancer. Eur J Surg Oncol. 2011;37:388-97. doi:10.1016/j.ejso.2011.01.018.

5. O'Brien ME, Wigler N, Inbar M, Rosso R, Grischke E, Santoro A, et al. Reduced cardiotoxicity and comparable efficacy in a phase III trial of pegylated liposomal doxorubicin $\mathrm{HCl}$ (CAELYX/ Doxil) versus conventional doxorubicin for first-line treatment of metastatic breast cancer. Ann Oncol. 2004;15:440-9.

6. Hulsermann U, Hoffmann MM, Massing U, Fricker G. Uptake of apolipoprotein E fragment coupled liposomes by cultured brain microvessel endothelial cells and intact brain capillaries. J Drug Target. 2009;17:610-8. doi:10.1080/10611860903105986.

7. Alexis F, Pridgen EM, Langer R, Farokhzad OC. Nanoparticle technologies for cancer therapy. Handb Exp Pharmacol. 2010;(197):55-86. doi:10.1007/978-3-642-00477-3_2

8. Lipinski CA, Lombardo F, Dominy BW, Feeney PJ. Experimental and computational approaches to estimate solubility and permeability in drug discovery and development settings. Adv Drug Deliv Rev. 2001;46:3-26.

9. Minchinton AI, Tannock IF. Drug penetration in solid tumours. Nat Rev Cancer. 2006;6:583-92. doi:10.1038/nrc1893.

10. Dennis MS, Jin H, Dugger D, Yang R, McFarland L, Ogasawara A, et al. Imaging tumors with an albumin-binding Fab, a novel tumortargeting agent. Cancer Res. 2007;67:254-61. doi:10.1158/00085472.CAN-06-2531.

11. Mier W, Hoffend J, Kramer S, Schuhmacher J, Hull WE, Eisenhut M, et al. Conjugation of DOTA using isolated phenolic active esters: the labeling and biodistribution of albumin as blood pool marker. Bioconjug Chem. 2005;16:237-40. doi:10.1021/bc034216c. 